\title{
Essentials of Glycobiology
}

\section{Third Edition}

Edited by Ajit Varki, University of California, San Diego, California, USA; Richard D. Cummings, Harvard Medical School, Boston, Massachusetts, USA; Jeffrey D. Esko, University of California, San Diego, California, USA; Pamela Stanley, Albert Einstein College of Medicine, New York, New York, USA; Gerald W. Hart, Johns Hopkins University, Baltimore, Maryland, USA; Markus Aebi, ETH Zurich, Zurich, Switzerland; Alan G. Darvill, University of Georgia, Athens, Georgia, USA; Taroh Kinoshita, Osaka University, Osaka, Japan; Nicolle H. Packer, Macquarie University, Sydney, Australia; James H. Prestegard, University of Georgia, Athens, Georgia, USA; Ronald L. Schnaar, Johns Hopkins University, Baltimore, Maryland, USA; Peter H. Seeberger, Max-Planck-Institute of Colloids and Interfaces, Potsdam, Germany

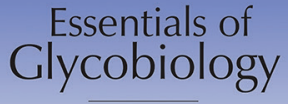

$\mathrm{D}$ efined in the broadest sense, Glycobiology is the study of the structure, biosynthesis, biology, and evolution of saccharides (sugar chains or glycans) that are widely distributed in nature in all living life-forms. Glycobiology is now one of the more rapidly growing fields in the natural sciences, with broad relevance to many areas of basic research, biomedicine, and biotechnology. The field includes the chemistry of carbohydrates, the enzymology of glycan formation and degradation, the recognition of glycans by specific proteins, roles of glycans in complex biological systems, and their analysis or manipulation by various techniques. The third edition of this primary textbook in the field continues in the prior tradition, seeking to provide a basic overview of Glycobiology, directed toward the advanced undergraduate or the beginning graduate-level student of molecular and cellular biology and biomedicine. Although efforts have been made to avoid a major increase in overall length of the text, substantial changes and improvements include the following:

- Broader focus on all lineages of life-forms in nature

- Wider range of topics, ranging from biology and medicine to chemistry and materials science

- Expanded international editorial board representing a wider range of expertise
- Wider range of contributing authors with expertise in specific areas

- Greatly expanded monosaccharide symbol nomenclature for the representation of glycans

- Greater attention to informatics, and the relevance to exploration of the glycome

\section{7, 823 pages, illustrated ( 223 color and $62 \mathrm{~B} \& \mathrm{~W}$ ), glossary, index}

Hardcover \$165 \$132 plus free shipping when you order at www.cshlpress.org/glycobiology ISBN 978-1-621821-32-8

\section{Advance Comments on the Third Edition of Essentials of Glycobiology}

"The field of glycobiology has matured. The comments of Nobel Laureates on the previous editions reflect the long-held belief that central functional roles played by the diversity of glycan chains would be revealed by research in this field. Now, as the result of advances in analytical chemistry and much deeper understanding of genomes, cell and tissue organization, this field has arrived. The third edition of Essentials of Glycobiology stands as the authoritative treatise on the subject, covering all aspects of the field and written by the world leaders in current research."

-James E. Rothman, Nobel Laureate in Medicine, 2013

"Difficult to analyze and synthesize artificially, glycans are often simply ignored. To do so is to avert one's gaze from an important part of life. More than mere decoration, glycans magnify the diversity of the already diverse molecules to which they are attached, affect protein folding and stability, direct traffic within cells, serve as signposts of self vs. non-self, create barriers that protect us, and conversely, defend microbes, making some of them the pathogens they are. It is hard to imagine a world without complex sugars, but if such a world existed, it would be much diminished. The third edition of Essentials of Glycobiology may be life changing for scientists who have not yet engaged with glycobiology, and will certainly be a treasured resource for those who already have."

-Bruce Beutler, Nobel Laureate in Medicine, 2011

wWW.cshlpress.org/glycobiology
"The importance of glycans has long been recognized and great advances have been reported on the synthesis and chemical analysis of this class of natural compounds. In my field, structural biology, carbohydrate moieties in glycoproteins and in complex multi-component macromolecular systems have been and continue to be difficult to handle. I greatly welcome the effort made in this multi-author volume to present results obtained with methods of structural biology in the context of the wealth of currently available chemical and biological data. I recommend the 3 rd edition of Essentials of Glycobiology as a highly useful reference on the current state of the field."

\section{—Kurt Wüthrich, Nobel Laureate in Chemistry, 2002}

"We think conventionally of the immune system as having evolved to deal with invading pathogens that express "foreign" proteins (and peptides), targeted via specific recognition units, particularly secreted antibodies and cell-bound $\mathrm{T}$ lymphocyte receptors. As the molecular revolution has unfolded, such science that relies on a reasonably direct correlation between genotype and phenotype has been relatively straightforward. Much more difficult to assess is the part played by glycosylation profiles in immune recognition and pathogen elimination. Perhaps even more intriguing is the issue of glycan abnormalities and recognition in cancer and many other disease processes. Now, in a third edition of Essentials of Glycobiology written by authoritative leaders in the field, we learn how this knowledge has been rapidly advancing, and see possibilities for real breakthroughs in understanding and therapy." 


\section{Essentials of Glycobiology \\ Third Edition}

\section{Contents}

\section{General Principles}

1. Historical Background and Overview

2. Monosaccharide Diversity

3. Oligosaccharides and Polysaccharides

4. Cellular Organization of Glycosylation

5. Glycosylation Precursors

6. Glycosyltransferases and Glycan-Processing Enzymes

7. Biological Functions of Glycans

8. A Genomic View of Glycobiology

\section{Structure and Biosynthesis}

9. N-Glycans

10. O-GalNAc Glycans

11. Glycosphingolipids

12. Glycosylphosphatidylinositol Anchors

13. Other Classes of Eukaryotic Glycans

14. Structures Common to Different Glycans

15. Sialic Acids and Other Nonulosonic Acids

16. Hyaluronan

17. Proteoglycans and Sulfated Glycosaminoglycans

18. Nucleocytoplasmic Glycosylation

19. The O-GlcNAc Modification

\section{Glycans in Evolution and Development}

20. Evolution of Glycan Diversity

21. Eubacteria

22. Archaea

23. Fungi

24. Viridiplantae and Algae

25. Nematoda

26. Arthropoda

27. Deuterostomes

\section{Glycan-Binding Proteins}

28. Discovery and Classification of Glycan-Binding Proteins

29. Principles of Glycan Recognition

30. Structural Biology of Glycan Recognition
31. R-Type Lectins

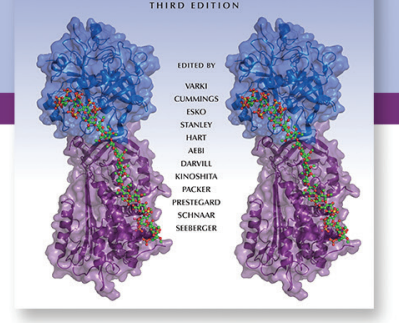

32. L-Type Lectins

33. P-Type Lectins

34. C-Type Lectins

35. I-Type Lectins

36. Galectins

37. Microbial Lectins: Hemagglutinins, Adhesins, and Toxins

38. Proteins That Bind Sulfated Glycosaminoglycans

Glycans in Physiology and Disease

39. Glycans in Glycoprotein Quality Control

40. Free Glycans as Signaling Molecules

41. Glycans in Systemic Physiology

42. Bacterial and Viral Infections

43. Parasitic Infections

44. Genetic Disorders of Glycan Degradation

45. Genetic Disorders of Glycosylation

46. Glycans in Acquired Human Diseases

47. Glycosylation Changes in Cancer

Methods and Applications

48. Glycan-Recognizing Probes as Tools

49. Glycosylation Mutants of Cultured Mammalian Cells

50. Structural Analysis of Glycans

51. Glycomics and Glycoproteomics

52. Glycobioinformatics

53. Chemical Synthesis of Glycans and Glycoconjugates

54. Chemoenzymatic Synthesis of Glycans and Glycoconjugates

55. Chemical Tools for Inhibiting Glycosylation

56. Glycosylation Engineering

57. Glycans in Biotechnology and the Pharmaceutical Industry

58. Glycans in Nanotechnology

59. Glycans in Bioenergy and Materials Science

60. Future Directions in the Glycosciences

Glossary, Study Guide, Index 